\title{
The use of a 3D-printed individualized navigation template to assist in the anatomical reconstruction surgery of the anterior cruciate ligament
}

\author{
Dejian Liu ${ }^{1}$, Yanlin $\mathrm{Li}^{1}$, Tao $\mathrm{Li}^{2}$, Yang Yu ${ }^{1}$, Guofeng Cai ${ }^{1}$, Guiran Yang ${ }^{1}$, Guoliang Wang ${ }^{1}$ \\ ${ }^{1}$ Department of Sports Medicine, The First Affiliated Hospital of Kunming Medical University, Kunming, China; ${ }^{2}$ Department of Trauma Surgery, \\ Affiliated Hospital of Yunnan University, Kunming, China \\ Contributions: (I) Conception and design: D Liu, Y Li, T Li, Y Yu; (II) Administrative support: Y Li; (III) Provision of study materials or patients: D \\ Liu, Y Li, Y Yu, G Cai, G Yang, G Wang; (IV) Collection and assembly of data: D Liu, G Yang; (V) Data analysis and interpretation: D Liu, Y Li; (VI) \\ Manuscript writing: All authors; (VII) Final approval of manuscript: All authors. \\ Correspondence to: Yanlin Li. Department of Sports Medicine, The First Affiliated Hospital of Kunming Medical University, 295 Xichang Road, \\ Kunming, China. Email: 1yl686373@126.com.
}

Background: To explore the location accuracy and early clinical outcomes of using a $3 \mathrm{D}$-printed
individualized navigation template to assist in the reconstruction of the anterior cruciate ligament (ACL).
Methods: A single center randomized control study was conducted. Patients with ACL injury were treated
with a conventional operation or an operation assisted by a $3 \mathrm{D}$-printed individualized navigation template (the
$3 \mathrm{D}$ group). The primary endpoint was the accuracy of the actual reconstruction compared with the planned
position.
Results: There were 20 and 23 participants in the conventional group and the $3 \mathrm{D}$ group, respectively.
There were no differences in the bone tunnel position between the actual postoperative position and
the preoperative design in the $3 \mathrm{D}$ group ( $\mathrm{P}>0.05)$. Compared with the $3 \mathrm{D}$ group, the positioning of the
femoral tunnel was more inferior and shallower in the conventional group $(\mathrm{P}<0.05)$. The position of the
tibia tunnel was closer to the anterior and medial edge of the tibial platform in the conventional group
compared to the $3 \mathrm{D}$ group $(\mathrm{P}<0.05)$. The intraoperative positioning time was shorter in the $3 \mathrm{D}$ group than
in the conventional group $(3.3 \pm 1.0 v s$. $5.9 \pm 1.8$ minutes, $\mathrm{P}<0.001)$. The Lysholm and International Knee
Documentation Committee scores did not differ between the two groups ( $>0.05$ for both), and all patients
improved after surgery $(\mathrm{P}<0.001)$.

Conclusions: The 3D-printed individualized navigation template showed good location accuracy and resulted in reduced intraoperative positioning time compared to the traditional method for ACL reconstruction.

Keywords: Printing; three-dimensional; precision medicine; spatial navigation; anterior cruciate ligament (ACL); arthroscopy

Submitted Oct 18, 2020. Accepted for publication Dec 01, 2020.

doi: $10.21037 /$ atm-20-7515

View this article at: http://dx.doi.org/10.21037/atm-20-7515

\section{Introduction}

Anterior cruciate ligament (ACL) rupture is a common injury in sports medicine (1-3). Increased physical activity and participation in various types of sports has escalated the incidence of ACL ruptures (1-3). Arthroscopic
ACL reconstruction is the main treatment for ACL ruptures (4). Recently, reconstruction after ACL rupture has changed from an isometric reconstruction to a more anatomical one (5). Biomechanical studies have shown that anatomical reconstruction can restore the rotational 
stability of the knee to near normal, and some clinical studies have demonstrated that anatomical reconstructions can achieve better outcomes than traditional isometric reconstructions (6-10).

With the introduction of precision medicine $(11,12)$, global sports medicine studies have begun to explore more accurate methods of reconstructing the ACL. Nevertheless, there are some differences in the location of the ACL insertion among different patients due to varying developmental conditions (13). Therefore, it is not easy to accurately determine the ACL anatomical insertion point during ACL reconstruction. Jaecker and colleagues (14) demonstrated that non-anatomical femoral tunnel positions were detected in $77.2 \%$ of the study cohort and non-anatomical tibial tunnel positions were found in $40.1 \%$ of patients. It has also been suggested that the successful placement of the anatomical bone tunnel is not solely dependent on whether the transtibial or anteromedial drilling techniques are used. Therefore, individualized ACL anatomical reconstruction is particularly important.

Computer-assisted surgery (CAS) can be applied in ACL reconstruction $(15,16)$. The use of computer-assisted navigation has been shown to assist in accurate positioning during ACL reconstruction surgery (17). Nevertheless, the clinical application of computer-assisted technologies for the accurate positioning of ACL reconstructions has rarely been reported. In this study, we developed an individualized 3D-printed navigation template for ACL reconstruction.

The aim of this study was to explore whether this novel technology could improve the location accuracy of the bone tunnel in ACL reconstruction surgery, and thereby improve clinical outcomes.

We present the following article in accordance with the CONSORT reporting checklist (available at http://dx.doi. org/10.21037/atm-20-7515).

\section{Methods}

\section{Patients}

Patients with ACL rupture who were admitted to the Department of Sports Medicine in the First Affiliated Hospital of Kunming Medical University between August 2018 and January 2019 were considered for this study.

Patients aged between 18-40 years old, who were diagnosed with an isolated ACL rupture and were compliant and cooperative were included in this study. Patients were excluded if they presented with injury to the meniscus or other ligament, systemic ligamentous laxity, severe knee deformity or flexion contracture deformity, or autoimmune diseases.

The 43 included participants were randomly divided into the control group or the experimental group using a computer-generated random number table. The participants in the control group were treated with the conventional operation, and those in the experimental group had a 3D-printed individualized navigation template to assist the operation. All participants underwent arthroscopic ACL reconstruction (using the hamstring tendon as the graft) by the same experienced chief physician who has 19 years of related experience.

\section{Preparation of the individualized navigation templates}

Under the premise that there is no reliable method of locating the ACL insertion position in patients with ACL rupture and the fact that the human limb structure is mostly symmetrical, this study reconstructed the injured ACL based on magnetic resonance imaging (MRI) images of the ACL and the bones on the healthy knee. Preoperatively, the bilateral knee joints were scanned with a 1.5-T MRI system (GE Healthcare, Waukesha, WI, USA) to obtain the three-dimensional fast spin echo (3D-FSE) sequence images of the knee joint. The obtained data were stored as Digital Imaging and Communications in Medicine (DICOM) files and imported into Mimics Research 19.0 (Materialise, Belgium) for image manipulation. The builtin 'Point Registration' method (image fusion technology) in the software was used to fuse the bilateral knee model with the axis as the benchmark. Then the 'Mirror' method was used to obtain the mirror image model of the healthy ACL, which was then used to simulate the affected ACL. We postulated that the intersecting points between this model and the affected knee bone were the insertion points of the affected ACL (Figure 1).

After obtaining the insertion points of the affected ACL, we saved the model in SLT (stereolithography) format, then imported it into Materialise Magics 21.0 (Materialise, Belgium) to design the navigation templates (Figure $2 A, B$ ). Finally, the navigation template model were imported into the medical image $3 \mathrm{D}$ reconstruction entity planning system [WASTON, model: ARP300A(B)] in the Gcode format. Polylactic acid (PLA) materials (non-toxic materials that can be safely used in clinical work) were used to print the model, which was then disinfected with lowtemperature plasma prior to clinical use. 

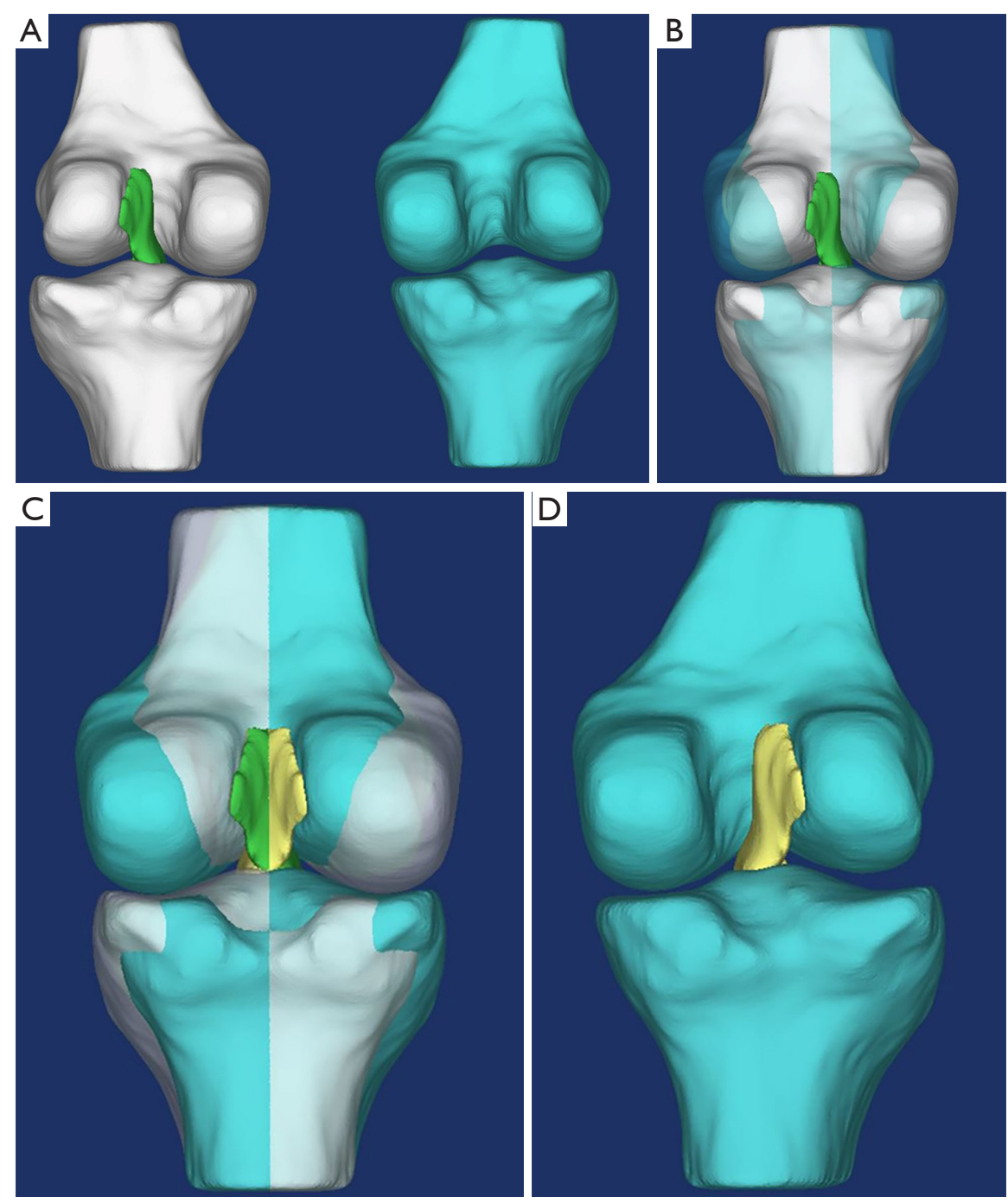

Figure 1 Construction of the affected ACL model using bilateral knee images (image fusion technology). (A) The 3D reconstruction model of the bilateral knee joints; (B) Point registration of the bilateral knee bones; (C) the healthy knee ACL mirror image was used to obtain a model of the affected ACL; (D) the affected bone and ACL model. 3D, three-dimensional; ACL, anterior cruciate ligament.

\section{Surgical methods}

Patients in the $3 \mathrm{D}$ group were administered routine anesthesia and ACL reconstruction aided by the $3 \mathrm{D}$-printed navigation template was performed using anteromedial (AM) techniques (18). On the femur side, the knee was bent at $90^{\circ}$. Under arthroscopy, the template was inserted into the intercondylar notch through the anteromedial portal. The medial end of the template was placed at the turning point of the lateral edge of the cartilage of the medial femoral condyle and the lateral surface of the medial condyle. The lateral end of the template was positioned at the medial surface of the lateral condyle. A 3-mm assistant port was used under the anteromedial portal. The tip of a piece of Kirschner wire (2 $\mathrm{mm}$ diameter) was placed into the guide hole through this assistant port. After drilling $5 \mathrm{~mm}$ into the femur, the template was removed. The knee was then bent at $120^{\circ}$, and the Kirschner wire was drilled through the lateral cortex of the lateral femoral condyle.

On the tibia side, the upper end of the template was inserted into the intercondylar notch through the anteromedial port. Then the lower end was gently rotated 

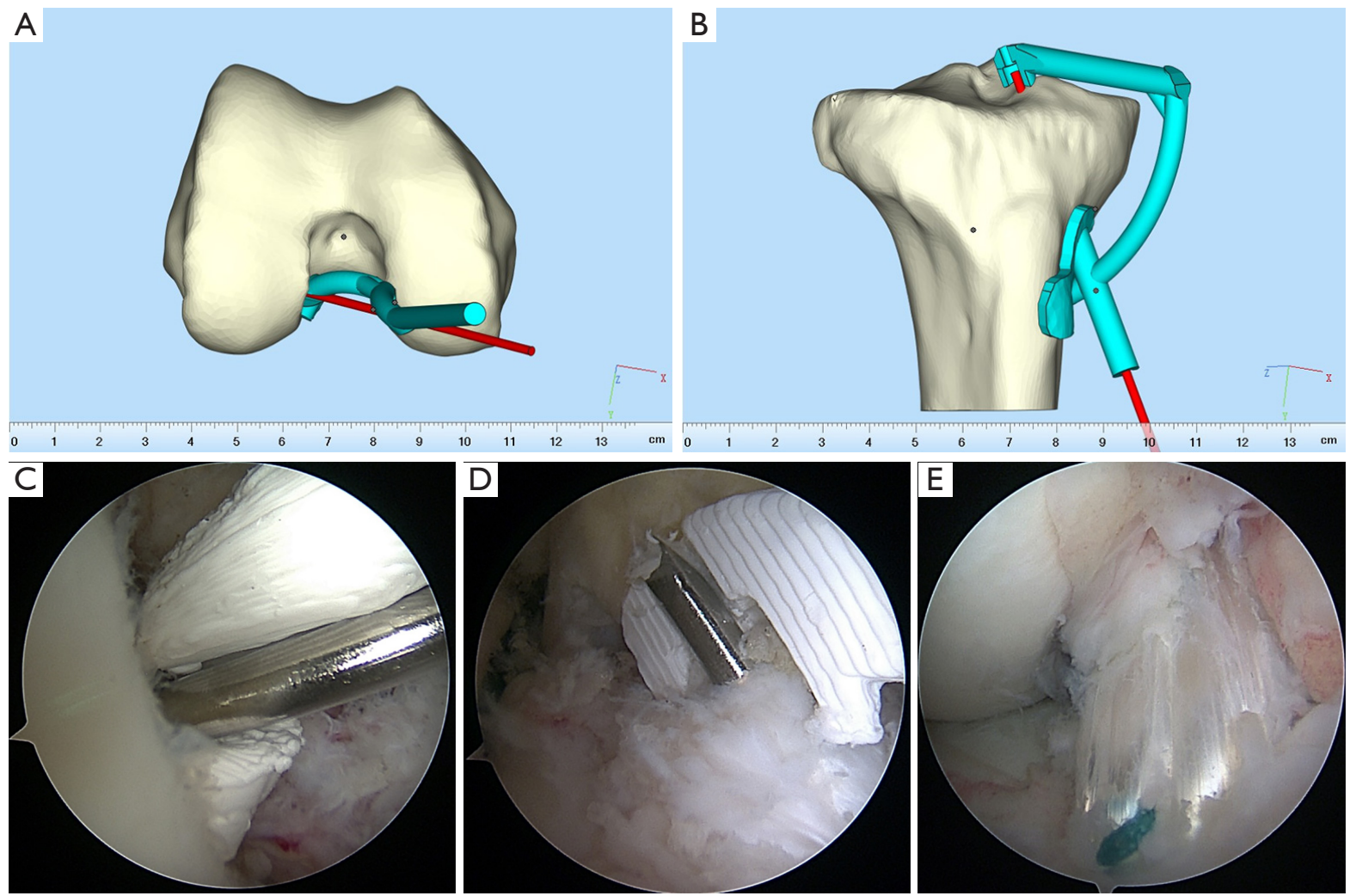

Figure 2 The design of the 3D-printed navigation template of the femur (A) and the tibia (B); arthroscopic views of the intraoperative use of the 3D-printed navigation template of the femur (C) and the tibia (D); arthroscopic view of the graft after reconstruction (E). 3D, threedimensional.

into the tendon incision, and the position was adjusted so that the navigation template fitted closely to the bony structure. A piece of Kirschner wire (2 $\mathrm{mm}$ diameter) was drilled through the guide hole under arthroscopy (Figure 2C,D).

After the tunnels were established, the graft was pulled through the tunnels. An Endobutton (Smith \& Nephew, London, United Kingdom) was used for femoral fixation. After tensioning the graft 20 times while the knee underwent cycling by an assistant, a biocompatible screw (Smith \& Nephew, London, United Kingdom) the same size as the tunnel was used for tibial fixation. A tibial fixation staple was also used. The position and tension of the graft were verified after reconstruction (Figure 2E).

The surgical procedure used for the conventional group was exactly the same as for the $3 \mathrm{D}$ treatment group, except without the use of a 3D-printed positioning tool.

\section{Analysis of the accuracy of the center point position of the bone tunnel}

The computed tomography (CT) data of the affected knee were collected at $1^{\text {st }}$ day post-operation, and a $3 \mathrm{D}$ model was constructed. The grid method $(19,20)$ was used to quantify the position of the center point of the actual bone tunnel compared with the intended position (Figure $3 A, B$ ). The accuracy of the position was the primary endpoint.

\section{Postoperative evaluations}

The function of the knee joint was investigated by the same rehabilitation physician for the $3 \mathrm{D}$ group and the conventional group. The Lysholm score and the International Knee Documentation Committee (IKDC) score were used to assess the affected knee before the operation, and at 6 and 12 months post-operation. X-ray 

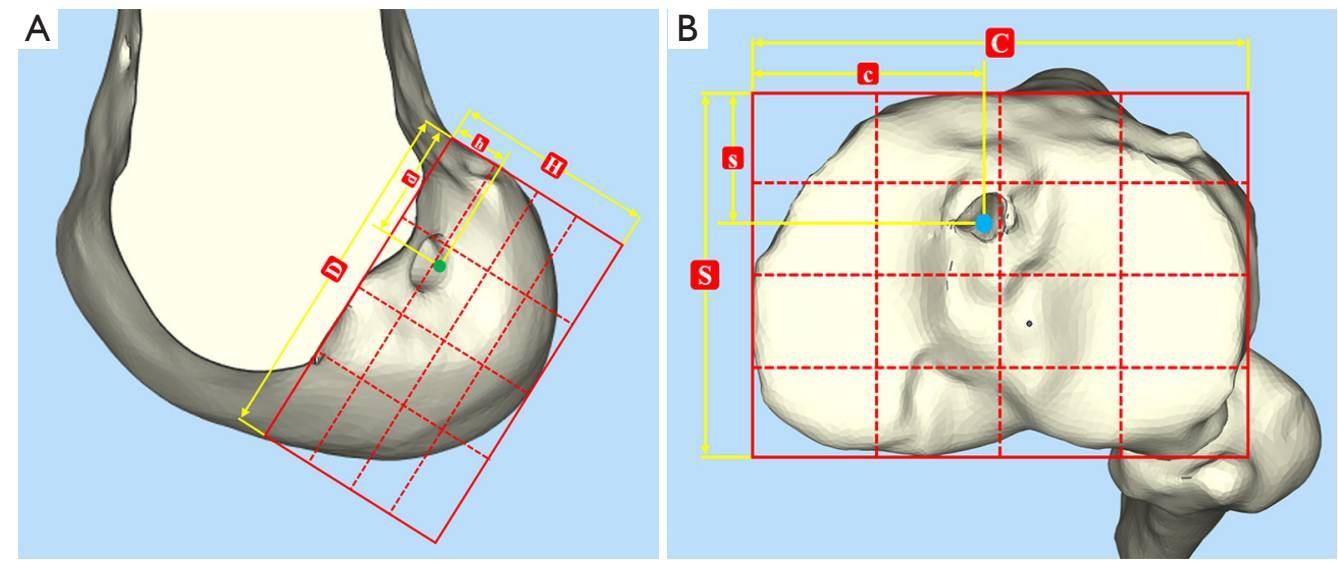

Figure 3 The grid method was used to quantify the position of the center point of the femoral tunnel (A) and the tibial tunnel (B).

and MRI of the affected knee were routinely performed after the operation.

\section{Statistical analyses}

Statistical analyses were performed using SPSS 19.0 software (IBM, Armonk, NY, USA). The categorical variables were presented as numbers (percentages) and compared using the chi-square test. The continuous variables that were normally distributed according to the KolmogorovSmirnov test were presented as mean \pm standard deviation. Data were analyzed using the independent-samples $t$-test (between groups), paired $t$-test (within groups), and analysis of variance (ANOVA) together with the Student-NewmanKeuls (SNK) post-hoc test (multiple within-group analyses). $\mathrm{P}$ values $<0.05$ were considered statistically significant.

\section{Ethical considerations}

All procedures performed in this study involving human participants were in accordance with the Declaration of Helsinki (as revised in 2013). This study (clinical trial registration number ChiCTR1900024005) was approved by the Medical Ethics Committee of The First Affiliated Hospital of Kunming Medical University [(2018) Ethics Audit L No. 21]. All patients provided written informed consent.

\section{Results}

\section{General data}

The complete follow-up rate was $100 \%(n=43)$ at 6 months post-operation and $95.3 \%$ at 12 months post-operation. The two participants who were lost to follow-up included one in the conventional group $(n=20)$ and one in the $3 \mathrm{D}$ group $(n=23)$. The average age of the participants was $26.0 \pm 4.1$ years. In the cohort, $56.1 \%$ were male and there was no statistical difference in the male to female ratio between the conventional group and the $3 \mathrm{D}$ group $(\mathrm{P}>0.05$, Table 1). Sports injury accounted for $87.8 \%$ of the cases. The 41 patients with complete follow-up data were included in the final analysis. The median follow-up was 14.1 months with a range of 12-15 months (Figure 4).

\section{Center point accuracy of the bone tunnel}

The distance between the center of the tunnel and the anatomic landmark was assessed according to the definitions used by Zantop and colleagues (21). There were no differences in the central points of the bone tunnels between the actual postoperative position and the preoperative design in the $3 \mathrm{D}$ group $(\mathrm{P}>0.05$, Table 2). The accuracy of the tunnel positioning was also assessed using an anatomical coordinate system to describe the location (Figure $5 A, B$ ). The position of the femoral tunnel in the conventional group was more inferior and shallower $(\mathrm{P}<0.001)$, and the position of the tibia tunnel was closer to the anterior and medial edge of the tibial platform $(\mathrm{P}<0.001$, Table 3) compared to the $3 \mathrm{D}$ group.

\section{Secondary endpoints}

The intraoperative positioning time was shorter in the $3 \mathrm{D}$ group compared to the conventional group $(3.3 \pm 1.0 \mathrm{vs}$. 
Table 1 Patient demographics

\begin{tabular}{|c|c|c|c|c|}
\hline Variable & All $(n=41)$ & Conventional group $(n=19)$ & $3 D$ group $(n=22)$ & $P$ \\
\hline Male & $23(56.1)$ & $11(57.9)$ & $12(54.5)$ & \\
\hline Female & $18(43.9)$ & $8(42.1)$ & $10(45.5)$ & \\
\hline Age (years) & $26.0 \pm 4.1$ & $26.8 \pm 4.5$ & $25.3 \pm 3.7$ & 0.220 \\
\hline Traffic accident & $5(12.2)$ & $2(10.5)$ & $3(13.6)$ & \\
\hline Sports injury & $36(87.8)$ & $17(89.5)$ & $19(86.4)$ & \\
\hline Knee, n (\%) & & & & 0.647 \\
\hline Left & $20(48.8)$ & $10(52.6)$ & $10(45.5)$ & \\
\hline Intraoperative positioning time (min) & $4.5 \pm 1.9$ & $5.9 \pm 1.8$ & $3.3 \pm 1.0$ & $<0.001$ \\
\hline
\end{tabular}

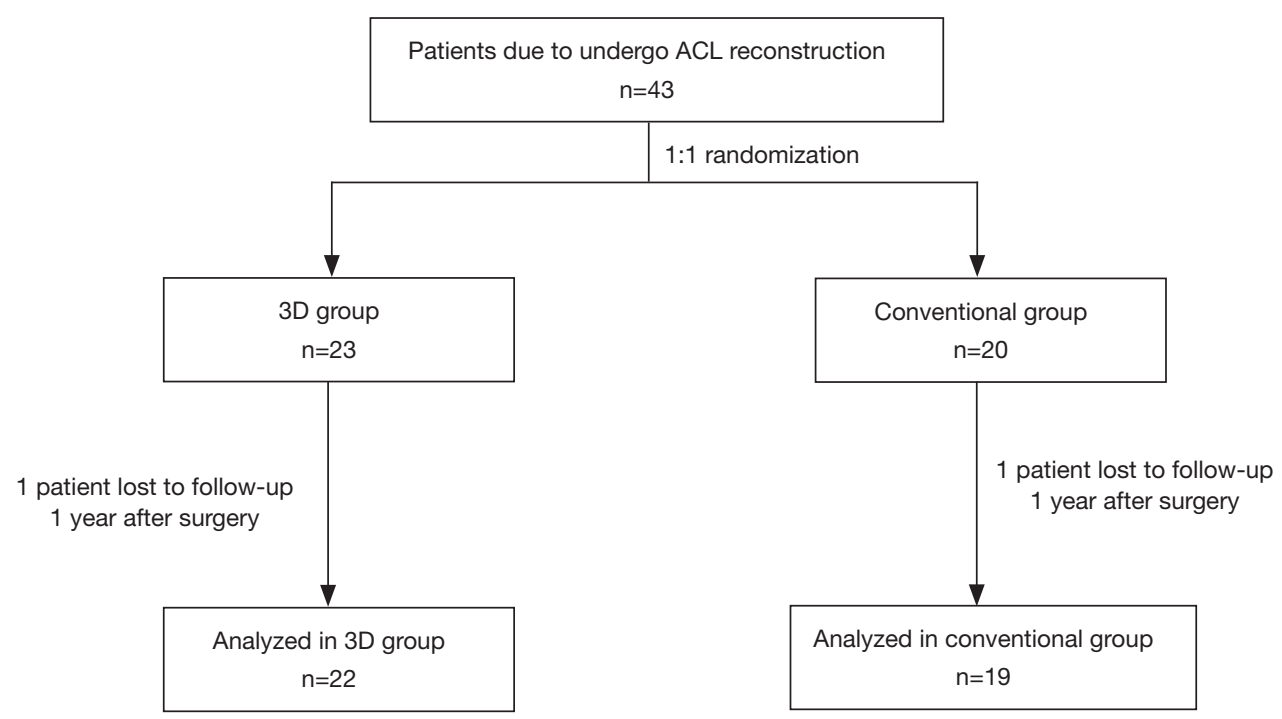

Figure 4 A flow diagram showing the inclusion of patients in the study. ACL, anterior cruciate ligament.

5.9 \pm 1.8 minutes, $\mathrm{P}<0.001$, Table 1). The Lysholm scores and IKDC scores at each time point postoperatively were significantly better than the preoperative scores in both groups ( $\mathrm{P}<0.001$ for all comparisons). However, there were no differences between the conventional group and the $3 \mathrm{D}$ group (Table 4). The range of motion for the affected knee returned to normal 12 months after the operation for both groups. The postoperative MRI scans revealed that none of the patients in either group showed signs of ligamentous laxity or other soft tissue injuries.

\section{Complications}

Incision swelling was not observed in any of the patients, and all participants achieved primary healing after the operation. There were no complications, such as vascular injury, deep vein thrombosis, and joint cavity infection. However, three patients (one in the conventional group 
Table 2 Comparison of the actual tunnel position with the expected position obtained from the model ( $\mathrm{n}=22)$

\begin{tabular}{lccc}
\hline Percentage of bone tunnel insertion point & Preoperative design & Postoperative actual position & \\
\hline Femoral tunnel & & & \\
Depth $(\mathrm{d} / \mathrm{D})(\%)$ & $27.8 \pm 4.4$ & $36.0 \pm 8.1$ & 0.651 \\
Height $(\mathrm{h} / \mathrm{H})(\%)$ & $36.4 \pm 8.6$ & & 0.643 \\
Tibial tunnel & & $38.1 \pm 2.2$ & 0.271 \\
Sagittal plane (s/S) (\%) & $38.6 \pm 1.6$ & $47.2 \pm 1.9$ & 0.265 \\
Coronal plane (c/C) (\%) & $47.5 \pm 1.7$ & \\
\hline
\end{tabular}

$\mathrm{d} / \mathrm{D}, \mathrm{h} / \mathrm{H}, \mathrm{s} / \mathrm{S}$ and $\mathrm{c} / \mathrm{C}$ can be seen in Figure $3 A$ and $B . \mathrm{d} / \mathrm{D}$, percentage of the depth of the femoral tunnel insertion point; $h / H$, percentage of the height of the femoral tunnel insertion point; $\mathrm{s} / \mathrm{S}$, percentage of the sagittal plane of the tibial tunnel insertion point; $\mathrm{c} / \mathrm{C}$, percentage of the coronal plane of the tibial tunnel insertion point.
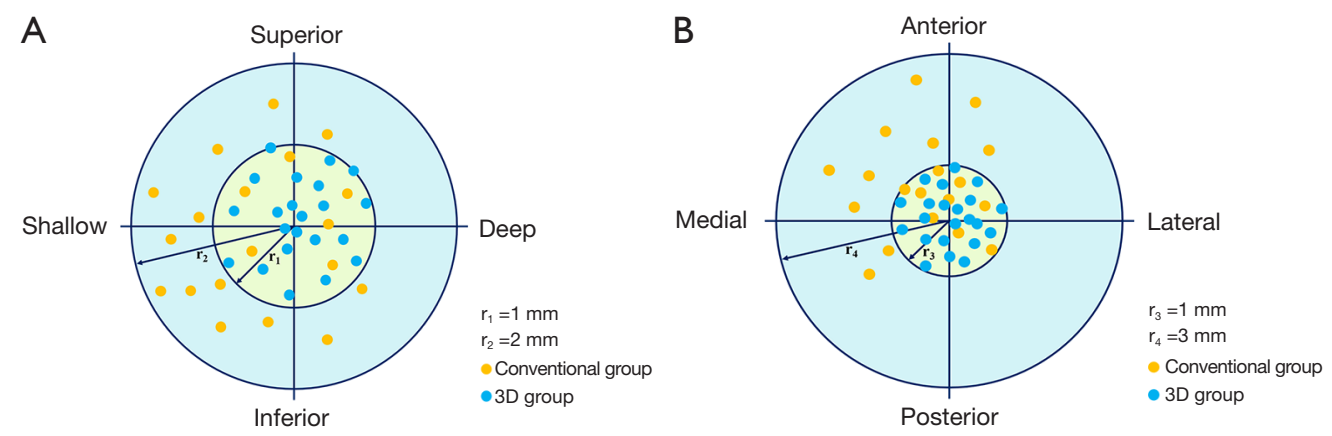

Figure 5 A comparison of the center point position of the femoral insertion point (A) and the tibial insertion point (B) in the 3D group and the conventional group.

Table 3 Comparison of post-operative tunnel position between two groups $(\mathrm{n}=41)$

\begin{tabular}{lccc}
\hline Percentage of bone tunnel insertion point & Conventional group $(\mathrm{n}=19)$ & 3D group $(\mathrm{n}=22)$ & \\
\hline Femoral tunnel & & & \\
Depth (d/D) (\%) & $30.4 \pm 3.7$ & $36.0 \pm 8.1$ & 0.025 \\
Height $(\mathrm{h} / \mathrm{H})(\%)$ & $40.7 \pm 6.4$ & & 0.045 \\
Tibial tunnel & & $38.1 \pm 2.2$ & 0.038 \\
Sagittal plane (s/S) (\%) & $36.5 \pm 2.6$ & $47.2 \pm 1.9$ & 0.010 \\
Coronal plane (c/C) (\%) & $45.3 \pm 2.5$ & \\
\hline
\end{tabular}

$\mathrm{d} / \mathrm{D}, \mathrm{h} / \mathrm{H}, \mathrm{s} / \mathrm{S}$, and $\mathrm{c} / \mathrm{C}$ can be seen in Figure $3 A$ and $B$. d/D, percentage of the depth of the femoral tunnel insertion point; $h / H$, percentage of the height of the femoral tunnel insertion point; $\mathrm{s} / \mathrm{S}$, percentage of the sagittal plane of the tibial tunnel insertion point; $\mathrm{c} / \mathrm{C}$, percentage of the coronal plane of the tibial tunnel insertion point. 
Table 4 Comparison of the knee joint function between the two groups $(n=41)$

\begin{tabular}{|c|c|c|c|}
\hline Function scores & Conventional group $(n=19)$ & $3 D$ group $(n=22)$ & $\mathrm{P}$ \\
\hline Pre-operation & $46.89 \pm 4.08$ & $46.68 \pm 4.25$ & 0.871 \\
\hline 6 months post-operation & $80.21 \pm 5.37$ & $81.16 \pm 4.23$ & 0.347 \\
\hline 12 months post-operation & $81.26 \pm 5.31$ & $82.00 \pm 4.39$ & 0.303 \\
\hline \multicolumn{4}{|l|}{ IKDC score } \\
\hline Pre-operation & $45.58 \pm 5.75$ & $45.86 \pm 5.15$ & 0.868 \\
\hline 6 months post-operation & $82.94 \pm 3.48$ & $83.91 \pm 3.15$ & 0.339 \\
\hline 12 months post-operation & $85.68 \pm 5.58$ & $87.23 \pm 5.60$ & 0.380 \\
\hline
\end{tabular}

IKDC, International Knee Documentation Committee scores.

and two in the 3D group) suffered from hypoesthesia of the infrapatellar nerve. Postoperatively, no adverse symptoms such as locking and limited extension were reported in the affected knee.

\section{Discussion}

ACL reconstruction surgery is complicated by anatomical variations among individuals $(13,14)$ and as such, CAS might be beneficial $(15,16)$. Therefore, the present study aimed to explore the benefits of using 3D-printed individualized navigation templates during ACL reconstruction surgery. The location accuracy and early clinical outcomes were examined. The data suggested that the 3D-printed individualized navigation templates resulted in improved location accuracy and shorter intraoperative positioning times compared to the traditional method of ACL reconstruction.

Accurately locating the ACL anatomical insertion point is challenging (14). Surgeons mainly locate this by using bone markers visualized under arthroscopy, but it is difficult to achieve a perfect ACL anatomical reconstruction, and the operation may not always be successful (22). Many researchers have explored novel methods to accurately locate the ACL insertion point, and found that using a fluoroscopy navigation system, a laser location system, or a reconstruction navigation system based on 2D$3 \mathrm{D}$ registration could achieve an accurate bone tunnel position (23-25). Ni and colleagues (26) used CT data to determine the ACL insertion location in 20 cases of cadaveric knee joints. This data was then applied to the design of a 3D-printed navigation template which was used to locate the bone tunnel position. They noted that the mean deviation of the actual position from the preoperative design was $0.57 \mathrm{~mm}$ for the femoral end and $0.58 \mathrm{~mm}$ for the tibial end. Despite the encouraging results, their navigation system necessitated an increased intraoperative radiation dose, and the procedure was relatively complex, thus limiting its clinical application and appeal. In addition, $\mathrm{Ni}$ and colleagues used relatively large bone anchors in the femur and tibia, and in clinical practice this may cause significant damage to the patients. Therefore, the results from this latter study do not have great clinical significance.

According to Zantop and colleagues (21), the anatomical femoral tunnel position in CT scan measurements should be within a range of $18.5-29.3 \%$ for depth and $22.3-53.6 \%$ for height, and the tibial tunnel position in the sagittal plane should be within a range of 30-44\%. Recently, Parkinson and colleagues (27) suggested that the central point of the normal ACL tibial insertion should be located $39 \%$ from the anterior and $48 \%$ from the medial side on a standardized tibial grid system. In our study, similar results were obtained using the grid method. In the preoperative design, the ACL femoral anatomical insertion was $27.8 \%$ in depth and $36.4 \%$ in height [compared with a median of $23.9 \%$ and $37.9 \%$, respectively, in studies by Zantop and colleagues (21)]. The ACL tibial anatomical insertion was located $38.1 \%$ from the anterior and $47.2 \%$ from the medial side of the tibia, in agreement with previous studies (27). This suggested that it is feasible to confirm 
the ACL insertion location of the affected knee using the methods adopted in this study. At the same time, there was no difference between the actual position of the bone tunnel center and the preoperative design used in the $3 \mathrm{D}$ group, suggesting that the navigation template designed in this study demonstrated good accuracy. When compared with the $3 \mathrm{D}$ group, the position of the femoral tunnel was more inferior and shallower in the conventional group, and the position of the tibia tunnel was closer to the anterior and medial edge of the tibial platform. The depth of the femoral insertion was outside the recommended range $(21,27)$. Therefore, we can infer that the $3 \mathrm{D}$ group might achieve a better ACL insertion position than the conventional group. The 'Mirror' method resulted in the individualized and precise determination of the position of the anatomical insertion point in ACL reconstruction surgery.

The use of $3 \mathrm{D}$ printing to produce individualized devices that assist in medical treatments is inexpensive and has many advantages, including shorter operation times, and improved accuracy of location or orientation (28-31). The present study demonstrated that a $3 \mathrm{D}$-printed navigation template could be used directly to locate the center point of the bone tunnel during the operation. This reduced the intraoperative positioning time required to determine the ACL insertion point compared to conventional methods. At 6 and 12 months after the operation, patients had better Lysholm and IKDC scores, suggesting that the function of the knee joint had recovered well. Patients did not present with limited knee extension during follow-up, which might indirectly indicate that the graft did not collide with the intercondylar notch, and the reconstructed bone tunnels were in a good position. In addition, no incision swelling, infection, or other discomforts were reported in the experimental group, indicating that the PLA materials used for the template in this study can be safely used in the clinical setting.

Once MRI data has been collected, the preparation of the individualized navigation templates takes about 6 hours. In the present study, the cost of the template was covered by the research project, but the estimated costs to the patients would be about 300 renminbi (RMB), or about 42 United States dollars for each patient. Hence, the low costs would justify the improvement in patient outcomes.

\section{Limitations}

This study was limited by its small sample size, which may have caused bias in the inclusion of the participants. Larger studies from multiple centers would be needed to confirm the results. A longer follow-up period would be beneficial to fully evaluate the outcomes and safety of using the $3 \mathrm{D}$ template. Due to the short follow-up period, only early outcomes could be assessed. In addition, there are also some limitations that should be considered when using the $3 \mathrm{D}$ template method. Firstly, an improper insertion angle of the navigation template and radian for the handle of the guide plate will affect the accuracy of the intraoperative location. Secondly, if the patient has an injury of the bilateral ACLs, the mirror image model cannot be generated as there is no healthy ACL, and thus, the accurate central position of the anatomical insertion of the affected ACL cannot be obtained. Hence, further research is needed regarding the design of the guide plate to allow an appropriate insertion angle. In addition, alternative designs methods will need to be explored for patients with bilateral ACL ruptures.

\section{Conclusions}

The use of the $3 \mathrm{D}$-printed individualized navigation templates designed in this study resulted in improved location accuracy and shorter intraoperative positioning time compared with the conventional method for ACL reconstruction surgery. Satisfactory early clinical outcomes were achieved after the operation, but further follow-up is required to explore its long-term outcomes.

\section{Acknowledgments}

The authors thank all the members of the research team for their contributions to this study.

Funding: The study was supported by the Yunnan Province Clinical Center for Bone and Joint Diseases (ZX201903-04), the Yunnan Chen Shiyi Expert Workstation (2018IC102), and the Yunnan Province Leading Talent Program (L-201601).

\section{Footnote}

Reporting Checklist: The authors have completed the CONSORT reporting checklist. Available at http://dx.doi. org/10.21037/atm-20-7515

Data Sharing Statement: Available at http://dx.doi. org/10.21037/atm-20-7515

Conflicts of Interest: All authors have completed the ICMJE 
uniform disclosure form (available at http://dx.doi. org/10.21037/atm-20-7515). The authors have no conflicts of interest to declare.

Ethical Statement: The authors are accountable for all aspects of the work in ensuring that questions related to the accuracy or integrity of any part of the work are appropriately investigated and resolved. All procedures performed in this study involving human participants were in accordance with the Declaration of Helsinki (as revised in 2013). This study (clinical trial registration number ChiCTR1900024005) was approved by the Medical Ethics Committee of The First Affiliated Hospital of Kunming Medical University [(2018) Ethics Audit L No. 21]. All patients provided written informed consent.

Open Access Statement: This is an Open Access article distributed in accordance with the Creative Commons Attribution-NonCommercial-NoDerivs 4.0 International License (CC BY-NC-ND 4.0), which permits the noncommercial replication and distribution of the article with the strict proviso that no changes or edits are made and the original work is properly cited (including links to both the formal publication through the relevant DOI and the license). See: https://creativecommons.org/licenses/by-nc-nd/4.0/.

\section{References}

1. Spindler KP, Wright RW. Clinical practice. Anterior cruciate ligament tear. N Engl J Med 2008;359:2135-42.

2. Cimino F, Volk BS, Setter D. Anterior cruciate ligament injury: diagnosis, management, and prevention. Am Fam Physician 2010;82:917-22.

3. Micheo W, Hernandez L, Seda C. Evaluation, management, rehabilitation, and prevention of anterior cruciate ligament injury: current concepts. PM R 2010;2:935-44.

4. Chen TZ, Wang YS, Li XS. Anterior cruciate ligament reconstruction using an anterior cruciate ligament stump. Wideochir Inne Tech Maloinwazyjne 2019;14:461-7.

5. Kim HS, Seon JK, Jo AR. Current trends in anterior cruciate ligament reconstruction. Knee Surg Relat Res 2013;25:165-73.

6. Tampere T, Devriendt W, Cromheecke M, et al. Tunnel placement in ACL reconstruction surgery: smaller intertunnel angles and higher peak forces at the femoral tunnel using anteromedial portal femoral drilling-a 3D and finite element analysis. Knee Surg Sports Traumatol Arthrosc
2019;27:2568-76.

7. Kilinc BE, Kara A, Oc Y, et al. Transtibial vs anatomical single bundle technique for anterior cruciate ligament reconstruction: A Retrospective Cohort Study. Int J Surg 2016;29:62-9.

8. Bedi A, Musahl V, Steuber V, et al. Transtibial versus anteromedial portal reaming in anterior cruciate ligament reconstruction: an anatomic and biomechanical evaluation of surgical technique. Arthroscopy 2011;27:380-90.

9. Shino K, Mae T, Tachibana Y. Anatomic ACL reconstruction: rectangular tunnel/bone-patellar tendonbone or triple-bundle/semitendinosus tendon grafting. J Orthop Sci 2015;20:457-68.

10. Arno S, Bell CP, Alaia MJ, et al. Does Anteromedial Portal Drilling Improve Footprint Placement in Anterior Cruciate Ligament Reconstruction? Clin Orthop Relat Res 2016;474:1679-89.

11. König IR, Fuchs O, Hansen G, et al. What is precision medicine? Eur Respir J 2017;50:1700391.

12. Blackstone EH. Precision medicine versus evidencebased medicine: individual treatment effect versus average treatment effect. Circulation 2019;140:1236-8.

13. Yahagi $Y$, Iriuchishima T, Horaguchi T, et al. The importance of Blumensaat's line morphology for accurate femoral ACL footprint evaluation using the quadrant method. Knee Surg Sports Traumatol Arthrosc 2018;26:455-61.

14. Jaecker V, Zapf T, Naendrup JH, et al. High non-anatomic tunnel position rates in ACL reconstruction failure using both transtibial and anteromedial tunnel drilling techniques. Arch Orthop Trauma Surg 2017;137:1293-9.

15. Dessenne V, Lavallee S, Julliard R, et al. Computerassisted knee anterior cruciate ligament reconstruction: first clinical tests. J Image Guid Surg 1995;1:59-64.

16. Zaffagnini S, Urrizola F, Signorelli C, et al. Current use of navigation system in ACL surgery: a historical review. Knee Surg Sports Traumatol Arthrosc 2016;24:3396-409.

17. Lee BH, Kum DH, Rhyu IJ, et al. Clinical advantages of image-free navigation system using surface-based registration in anatomical anterior cruciate ligament reconstruction. Knee Surg Sports Traumatol Arthrosc 2016;24:3556-64.

18. Shafizadeh S, Jaecker V, Otchwemah R, et al. Current status of ACL reconstruction in Germany. Arch Orthop Trauma Surg 2016;136:593-603.

19. Bernard M, Hertel P, Hornung H, et al. Femoral insertion of the ACL. Radiographic quadrant method. Am J Knee Surg 1997;10:14-21; discussion 22. 
20. Tsukada H, Ishibashi Y, Tsuda E, et al. Anatomical analysis of the anterior cruciate ligament femoral and tibial footprints. J Orthop Sci 2008;13:122-9.

21. Zantop T, Wellmann M, Fu FH, et al. Tunnel positioning of anteromedial and posterolateral bundles in anatomic anterior cruciate ligament reconstruction: anatomic and radiographic findings. Am J Sports Med 2008;36:65-72.

22. van Eck CF, Schkrohowsky JG, Working ZM, et al. Prospective analysis of failure rate and predictors of failure after anatomic anterior cruciate ligament reconstruction with allograft. Am J Sports Med 2012;40:800-7.

23. Moloney G, Araujo P, Rabuck S, et al. Use of a fluoroscopic overlay to assist arthroscopic anterior cruciate ligament reconstruction. Am J Sports Med 2013;41:1794-800.

24. Yuan Z, Bian N, Hao Y, et al. Laser-guided transtibial technique improved single-bundle reconstruction of anterior cruciate ligament. J Orthop Surg Res 2018;13:184.

25. Guo N, Yang B, Ji X, et al. Intensity-based 2D-3D registration for an ACL reconstruction navigation system. Int J Med Robot 2019; 15:e2008.

26. Ni J, Li D, Mao M, et al. A Method of Accurate Bone Tunnel Placement for Anterior Cruciate Ligament Reconstruction Based on 3-Dimensional Printing Technology: A Cadaveric Study. Arthroscopy

Cite this article as: Liu D, Li Y, Li T, Yu Y, Cai G, Yang G, Wang G. The use of a 3D-printed individualized navigation template to assist in the anatomical reconstruction surgery of the anterior cruciate ligament. Ann Transl Med 2020;8(24):1656. doi: 10.21037/atm-20-7515
2018;34:546-56.

27. Parkinson B, Gogna R, Robb C, et al. Anatomic ACL reconstruction: the normal central tibial footprint position and a standardised technique for measuring tibial tunnel location on 3D CT. Knee Surg Sports Traumatol Arthrosc 2017;25:1568-75.

28. Lim CG, Campbell DI, Cook N, et al. A case series of rapid prototyping and intraoperative imaging in orbital reconstruction. Craniomaxillofac Trauma Reconstr 2015;8:105-10.

29. Park SW, Choi JW, Koh KS, et al. Mirror-Imaged Rapid Prototype Skull Model and Pre-Molded Synthetic Scaffold to Achieve Optimal Orbital Cavity Reconstruction. J Oral Maxillofac Surg 2015;73:1540-53.

30. Moser N, Santander P, Quast A. From 3D imaging to 3D printing in dentistry - a practical guide. Int J Comput Dent 2018;21:345-56.

31. Sieira Gil R, Roig AM, Obispo CA, et al. Surgical planning and microvascular reconstruction of the mandible with a fibular flap using computer-aided design, rapid prototype modelling, and precontoured titanium reconstruction plates: a prospective study. Br J Oral Maxillofac Surg 2015;53:49-53.

(English Language Editor: J. Teoh) 